\title{
Maintenance therapy in ovarian cancer: Molecular basis and therapeutic approach (Review)
}

\author{
MONICA BINASCHI ${ }^{1}$, CECILIA SIMONELLI ${ }^{2}$, CRISTINA GOSO $^{1}$, \\ MARIO BIGIONI $^{1}$ and CARLO ALBERTO MAGGI ${ }^{3}$ \\ ${ }^{1}$ Department of Pharmacology, Menarini Ricerche, Pomezia, Rome; ${ }^{2}$ Clinical Research Menarini Ricerche; \\ ${ }^{3}$ Department of Pharmacology, Menarini Ricerche, Florence, Italy
}

Received October 14, 2010; Accepted January 3, 2011

DOI: $10.3892 / \mathrm{etm} .2011 .192$

\begin{abstract}
Ovarian cancer has the highest mortality rate among gynaecological tumours despite the fact that the majority of patients with advanced disease achieve complete remission after first-line surgery and chemotherapy. Unfortunately, disease recurrence occurs in the majority of patients and second-line treatments are not curative. Clearly, the persistence of dormant and drug-resistant cells after front-line treatments results in the inability to cure the disease. The identification of cancer-initiating cells or cancer stem cells as key players in the development of recurrence has opened up a novel field of research aimed at identifying additional innovative therapeutic approaches. Strategies of maintenance therapy to extend the survival of patients have been studied, but to date no overall survival benefit has been detected. Currently, numerous clinical trials have just been completed or are ongoing involving patients achieving a complete clinical response after first-line chemotherapy in order to evaluate the efficacy of different therapeutic approaches in terms of disease-free survival and overall survival. At the 2010 ASCO meeting, the first positive results of a phase III clinical trial in this setting were presented: bevacizumab ( $15 \mathrm{mg} / \mathrm{kg}$ i.v. every 21 days) added to first-line chemotherapy and continued for an additional 15 cycles was found to prolong progression-free survival of 3.8 months in comparison to 6 cycles of chemotherapy alone or only 6 cycles of chemotherapy plus bevacizumab. In addition, positive results were announced for a second phase III trial testing bevacizumab in the same setting, but at half dose. The final assessment of the overall clinical benefit and the approval of bevacizumab in maintenance therapy by regulatory agencies is expected to be positive, as are the final results of abagovomab phase III trial MIMOSA, another antibody-based therapy tested as a maintenance treatment for advanced ovarian cancer
\end{abstract}

Correspondence to: Dr Monica Binaschi, Department of Pharmacology, Menarini Ricerche, Via Tito Speri 10, I-00040 Pomezia, Rome, Italy

E-mail: mbinaschi@menarini-ricerche.it

Key words: ovarian cancer, maintenance therapies, CA-125, abagovomab patients. Encouraging preliminary results confirming the safety profile and the immunogenic activity of abagovomab were presented at the last ASCO meeting. The final results are expected to be released in the first half of 2011.

\section{Contents}

1. Introduction

2. Basis of ovarian cancer recurrence: Cancer stem cells

3. Maintenance therapy approaches in ovarian cancer

4. Conclusion

\section{Introduction}

Ovarian cancer is the most lethal gynaecological cancer. It is the fifth leading cause of cancer-related mortality among women both in Europe and the US. The majority (90\%) of ovarian cancers arise from the surface epithelium, and tumourigenesis has been associated with inflammation and wound repair during ovulation.

Approximately $75 \%$ of ovarian cancer patients have advanced disease at the time of initial diagnosis, since early symptoms are common to many gastrointestinal and genitourinary conditions; indeed, ovarian cancer is referred to as the 'silent killer'. If ovarian cancer is identified at an early stage, when the disease is confined to the ovary, the survival rate improves, since it is highly treatable. In early stage disease (FIGO stage IA-IIA), surgery alone is curative. However, early diagnosis is rare due to the absence of a screening test. To date, screening tests such as CA125 levels and exams such as transvaginal sonography to detect early ovarian cancer have not provided satisfactory results, thus more sensitive and more specific assays are required $(1,2)$.

Recently, a randomised controlled trial (United Kingdom Collaborative Trial of Ovarian Cancer Screening) (Trial ISRCTN22488978 and NCT00058032) that will end in late 2014 was designed to assess the effect of screening on mortality. Post-menopausal women $(202,638)$ were randomly assigned to groups: no treatment, annual CA125 screening interpreted with a risk algorithm plus transvaginal ultrasound (multimodal screening) and annual screening with transvaginal 
ultrasound. The results of the effect of screening on mortality are expected in 2014 (3).

New efforts have emerged to identify new serum markers. Although many have acceptable specificity, the majority do not. Recently, a test using six biomarkers, developed by Yale University, was marketed by LabCorp (OvaSure) as an ovarian cancer screening test for high-risk populations (4). Unfortunately, both scientific communities, the Society of Gynecologic Oncology and the FDA, agreed that the interpretation of the results on which the test is based was premature and additional research is required to validate the test.

Of note, the clinical utility of a screening test is clear if it improves survival among screened women. Therefore, due to the low prevalence of ovarian cancer, the survival end point would require large-scale studies. Nevertheless, it is possible that through the integration of genomic and proteomic databases for biomarker discovery, promising molecules will be identified and validated (6).

The standard first-line treatment for patients with metastatic disease is debulking surgery and platinum/taxane-based chemotherapy. Surgery is a crucial part of ovarian cancer treatment, and patients operated on by a specialised surgeon were found to have a better overall survival (OS) (7-9). Optimal debulking surgeries have been correlated with increased survival rates, and surgical staging has also been shown to play a key role in the management of ovarian cancer patients. Indeed, ovarian cancer is one of the few tumours in which cytoreductive surgery is carried out even when complete resection is impossible. Thus, the experience of the surgeon and the institution affects the survival rate of the patients. Despite this, many women with ovarian cancer do not have their initial surgery performed by a gynaecological oncologist. For this reason it is crucial to determine whether or not a pelvic mass likely represents a malignancy.

Recently, the FDA has approved the first multiprotein ovarian cancer test to determine whether pelvic masses are cancerous. The test, called OVA1, combines CA125, $\beta 2$-microglobulin, tranferrin, apolipoprotein A1 and transthyretin associated with a proprietary algorithm. It is an ovarian triage test that, combined with clinical assessement, has a greater than $90 \%$ sensitivity and a $90 \%$ negative predictive value for women with planned surgery (10). A rival test is being developed by Fujirebio Inc. based on CA125 and HE4 using a risk of malignancy algorithm called ROMA (11).

It is now well-established that platinum/taxane-based chemotherapy improves both OS and progression-free survival (PFS). In the past 30 years, different classes of drugs in various combinations have been evaluated in a number of randomised controlled studies, showing that in front-line treatment the combination of platinum and paclitaxel yields a response rate greater than $80 \%$ in advanced disease (12). Nevertheless, the period of clinical responses is short, and the majority of patients experience disease recurrence and drugresistance.

\section{Basis of ovarian cancer recurrence: Cancer stem cells}

Despite the initial effectiveness of primary therapy against advanced stage ovarian cancer, the majority of these cases recur months to years following diagnosis. The cause of disease recurrence may involve cancer-initiating cells (CICs) that survive chemotherapy and enter a period of dormancy while residing in as yet undefined niches within the body before being triggered to initiate renewed growth.

Moreover, recurrent disease is no more sensitive to chemotherapy, and it is possible that this feature is due to the presence of CICs also termed cancer stem cells (CSCs). The CSC hypothesis claims the presence of a rare population of progenitor cells with unlimited regenerative capacity within a tumour. These cells, identified in many solid tumours (13), have been described in ovarian cancer cell lines as well as in patient samples (14-19). CICs are capable of self-renewal, and during asymmetric division are able to generate two types of cell: the first one is characterised by stem cell-like features, and the other is committed to differentiation, thus generating the heterogeneity usually found in tumours. The evidence for the existence of CICs in ovarian cancers has been based on their ability to form tumours sooner than bulk tumour cells when inoculated in immunodeficient mice. These subsets of cells have been, in various studies, characterised by the expression of CD133, CD44 and CD117 or by the ability to exclude Hoechst dye (SP cells), as these cells also express high levels of various types of membrane-spanning ATP binding cassette transporters, such as multidrug-resistant gene 1 and breast cancer-resistance protein 1 (15-19). The potential overlap or independence of subpopulations characterised by the expression of CD44, CD133 or side population phenotype (SP cells) has yet to be evaluated.

These candidate ovarian CSCs are not only chemoresistant to conventional chemotherapies, including doxorubicin, cisplatin and paclitaxel, but it has also been suggested that they proliferate in the presence of antiblastic drugs (15). Thus, the increased chemoresistance of CICs may allow them to survive through primary therapy leading to disease recurrence (20).

In this scenario, a number of therapeutic approaches have been evaluated in order to prolong the disease-free period and OS, unfortunately to date these have been unsuccessful (Table I). Other novel therapies have been recently tested or are being evaluated in ongoing clinical trials (Table II). These approaches specifically target metastatic cancer cells which survived the first-line therapy, and this could be supported, once available, by approaches aimed to specifically destroy CICs. The latter may represent a crucial tool for concomitant supplementation or support of maintenance therapy. A number of studies are ongoing to identify potential intracellular targets, the inhibition of which may block CIC proliferation.

CICs have a unique molecular profile characterised by constitutive NF- $\mathrm{KB}$ activity, and the secretion of pro-inflammatory cytokines may serve as growth factors and enhance resistance to apoptosis (15). In agreement, the pharmacological inhibition of NF- $\mathrm{\kappa B}$ by Riocalyxim B-induced caspase activation decreased cytokine production and induced cell death in ovarian CSCs (21). Another possibility to attack this subpopulation of cancer cells has been recently reported in vitro (19); treatment with interferon (INF)- $\alpha$ caused a reduction in SP cell content in various ovarian cancer cell lines. However, a randomised phase III trial showed no benefit for interferon maintenance in terms of either OS or clinical event-free survival (22). It has also been reported that the phenotype of cancer progenitor cells depends on epigenetic alteration, 


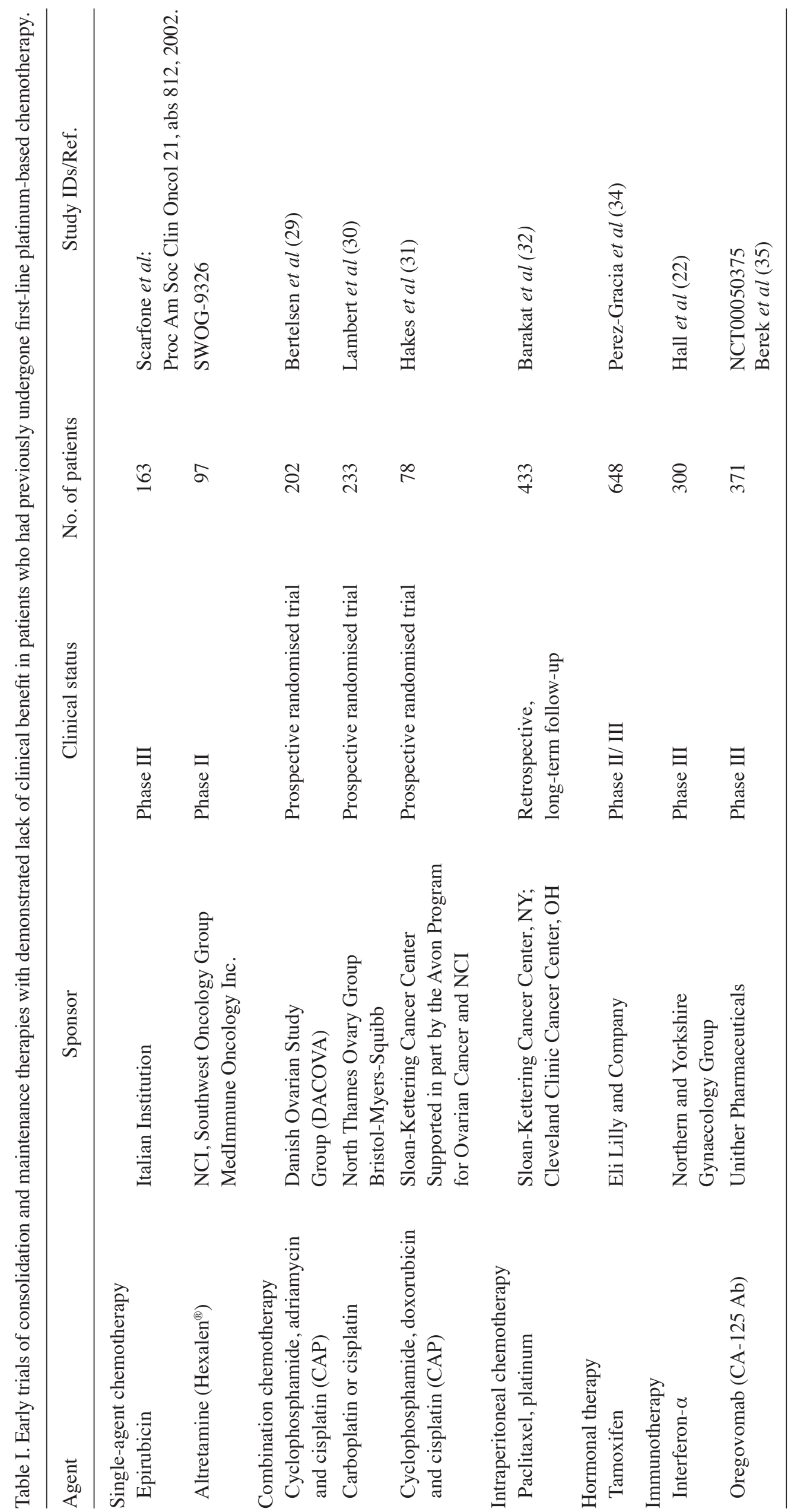




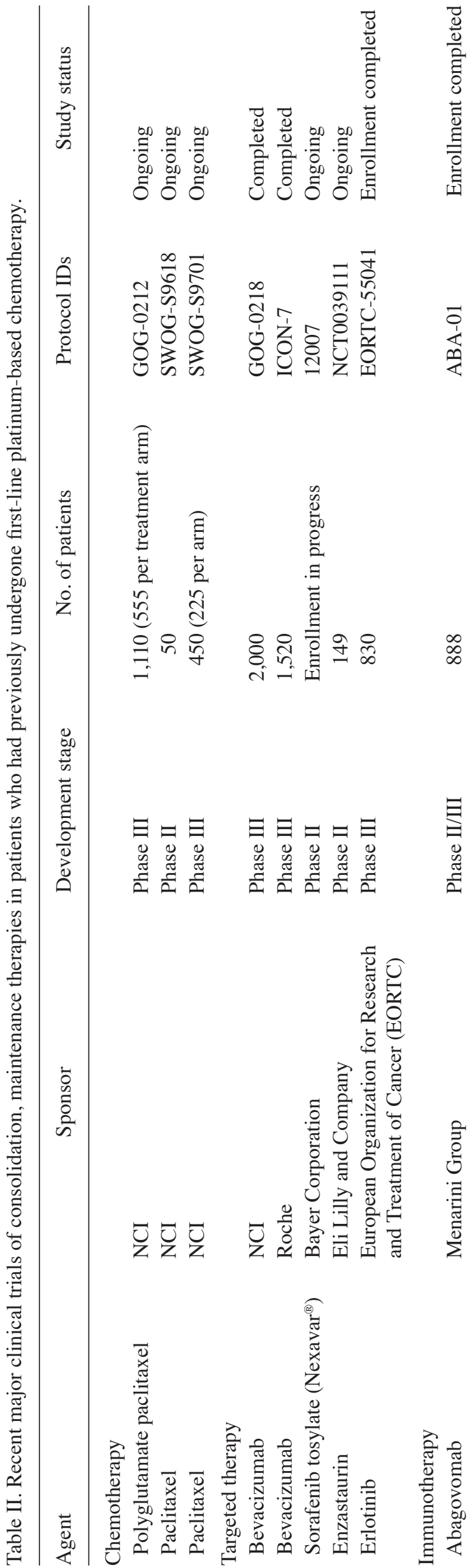

including DNA methylation and histone modifications $(23,24)$. In particular, the expression of CD133, the surface marker that demarcates the ovarian cancer initiating population, but whose function is currently unknown, is directly regulated by epigenetic modifications (18).

Recent findings with therapeutic implications have suggested that tumour suppressors play a role in the quiescent state of CICs which is the cause of resistance to conventional and targeted therapies. Thus, the transient pharmacological inhibition of tumour suppressors leads to the cycling of CICs, resulting in the impairment and exhaustion of CICs and possibly representing a new therapeutic opportunity (25). This model is currently being tested in clinical trials in CML and prostate cancer.

The recurrence of ovarian cancer has also been ascribed to Notch 3 overexpression (26). Amplification of the Notch3 locus has been observed in ovarian cancer with a worse clinical outcome. Noch3 expression also increases the expression of a number of genes associated with embryonic stem cells and leads to a decrease in carboplatin sensitivity. Thus, it has been suggested that the Notch3 pathway reprograms tumour cells towards chemoresistance (26).

The interaction of cancer cells with the microenviroment is also crucial, since mesenchymal stem cells play a role in the development of ovarian cancer metastasis and resistance to treatment. Tumour-associated mesenchymal cells co-cultured with ovarian cancer cells are able to secrete factors, including CXCL12 inducing thermal tolerance (27). Therefore, targeting the interaction between stromal and cancer cells through the inhibition of CXCL12/CXCR4 interaction may improve the sensitivity of ovarian cancer to hyperthermia, benefitting hyperthermia intraperitoneal chemotherapy.

\section{Maintenance therapy approaches in ovarian cancer}

Maintenance therapy, targeting residual subclinical disease, has been explored as a strategy for improving patient survival in ovarian cancer.

Early trials. In the past, numerous approaches were tested in this setting, i.e, prolongation of chemotherapy, hormonal therapy, targeted therapy and immunotherapy (Table I). The first trials were mainly based on prolonged administration of single-agent chemotherapy (28), extended cycles of combination chemotherapy (29-31) or intraperitoneal chemotherapy (32). The early trials provided negative results, with no prolongation of OS. The GOG 178 trial, which tested the efficacy of prolonged taxol administration (12 additional cycles vs. 3 additional cycles), showed a clinically longer PFS in the arm treated with 12 cycles, but, similar to previous trials, did not impact OS. The recent update of this study still confirmed the absence of an effect on OS. Numerous reasons can be ascribed to the lack of effect on OS: crossover of patients of the 3 cycle arm to longer treatment, a sample size insufficient to detect the difference or second-line treatment that equalised the outcome. Furthermore, the prolongation of taxol administration gives rise to concerns about the tolerability of long and potentially toxic treatment in comparison to the limited clinical benefit obtained.

During the past few years, hormonal therapy has been considered as a possible option for maintenance treatment, but 
tamoxifen has not been found to extend survival in patients with advanced ovarian cancer. However, few trials were randomised controlled trials, and the possible role of tamoxifen may have been underestimated (34).

Immunotherapy has been tested with two approaches: by boosting an aspecific immune host response or by trying to elicit specific response through immunization with tumourassociated antigens as specific targets.

Indeed the 'aspecific' approach was found to be ineffective as demonstrated by the INF- $\alpha$ trial, which found no improvement in the survival of patients with epithelial cancer following surgery and/or chemotherapy, and no benefit for interferon maintenance therapy was noted in terms of either OS or disease-free survival (DFS) (22).

More important is the 'specific' immune approach, which aims to produce an immune response against cells expressing specific tumour-associated antigens (TAAs). These molecules, even if overexpressed in cancer cells, are traditionally weakly immunogenic, as the large majority of these in humans are non-mutated self antigens.

CA125, also known as MUC16, is a large membraneassociated mucin protein which is overexpressed in more than $80 \%$ of epithelial ovarian cancers. The soluble molecule secreted in patient blood is used as a marker for tumour identification and/or progression. Due to its poor immunogenicity, the host organism is not able to mount an adequate immune response against it.

CA125, a TAA, has been previously used as a target of oregovomab, a murine monoclonal antibody, able to directly recognise and bind it. A direct effect of oregovomab on CA125-positive tumour cells has not been demonstrated, and the induction of a specific immune response has been described as the main mechanism of action. Oregovomab provided some promising results in a phase II study, where efficacy in a specific subpopulation of patients with an optimal prognosis, was indicated (39). However, it failed to demonstrate efficacy as maintenance immunotherapy after front-line therapy in a randomised double blind phase III clinical trial (35).

Recent trials and results. An improvement in PFS remains the aim of recent clinical research in this setting, however concerns derived from previous studies are the toxicity and the consequent impact of maintenance regimens on the quality of life of patients (36). Therefore, recent research has focused on the development of novel antiblastic drugs/regimens with limited toxicity, such as polyglutamate paclitaxel, or regimens, including agents targeting alternative pathways, i.e., VEGF inhibition pathway, EGFR, TK, mTOR inhibitors and on boosting the host immune response. The main clinical trials which are ongoing or have just been completed, investigating maintenance therapy after first-line chemotherapy in ovarian cancer, are documented in Table II.

Chemotherapy approaches. To date, prolonged administration of paclitaxel has been the only therapeutic approach which has shown a significant benefit on PFS. Based on this finding, the phase III GOG-212 trial is currently evaluating the effect of a new taxane derivative: prolonged administration of the new drug (12 cycles) vs. a prolonged administration of paclitaxel (12 cycles). The new taxane is expected to overcome resis- tance and have a better safety profile. The latter characteristic renders the prolonged administration of the new derivative more tolerable in comparison to paclitaxel. Another tested hypothesis deals with assessing the feasibility of the paclitaxel regimens; in fact, two trials are comparing weekly administration vs. the 3-week interval (Table II).

Targeted approaches. The VEGF pathway is a promising target for the treatment of advanced ovarian cancer. It has been previously reported that VEGF is expressed in $90 \%$ of human ovarian tumours (37-39) and that high levels of VEGF found in the serum, plasma and ascites of ovarian cancer patients are associated with poor prognosis (40). In pre-clinical studies, it was revealed that inhibition of VEGF activity inhibited ovarian tumours in an athymic mouse model (41). Moreover, a pre-clinical report revealed that bevacizumab used in maintenance therapy after a complete response to cisplatin plus bevacizumab in a murine xenograft model significantly delayed or inhibited the regrowth of ovarian cancer and prolonged survival (42), thus supporting the rationale for the clinical trials.

Bevacizumab has demonstrated its efficacy as a singleagent $(43,44)$ and in combination (39) for the treatment of recurrent ovarian cancer. Some serious toxicity was noted; in particular, the Genentech Study (44) was closed early due to a high rate of spontaneous bowel perforations (43-46). Recently, two large clinical trials, GOG-218 and ICON-7, which evaluated the benefit of bevacizumab in combination with first-line chemotherapy and as maintenance therapy, have been completed. GOG-218 enrolled more than 1,800 stage III-IV ovarian cancer patients in 3 arms. In the first arm, patients were treated with 6 cycles of chemotherapy which included carboplatin plus paclitaxel (CP) plus 15 cycles of a placebo. In the second arm, they were treated with 6 cycles of $\mathrm{CP}$ plus bevacizumab (15 mg/kg i.v.) plus 15 additional placebo cycles. In the third arm, patients were treated with 6 cycles of $\mathrm{CP}$ plus bevacizumab and subsequently with 15 additional bevacizumab cycles every 21 days. The results were presented at the last ASCO meeting. Bevacizumab showed a significantly longer PFS (3.8 months) when administered concomitantly with $\mathrm{CP}$ and thereafter for 15 additional cycles, while the concomitant use of bevacizumab and CP (arm 2) did not improve PFS in comparison to chemotherapy alone (arm 1) (Burger et al: J Clin Oncol 28 (Suppl), abs. LBA1, 2010).

Bevacizumab is the first targeted compound able to improve PFS in the maintenance setting in advanced ovarian cancer. However, data on OS are still pending. Moreover, concerns have arisen concerning the duration and high cost of treatment. Another phase III trial (ICON-7), testing bevacizumab as a mainteinance therapy in ovarian cancer, has just been completed, and positive results have been announced by the sponsor (Pfisterer et al: IGCS, abs. 805, 2010). It is worth noting that in ICON-7, bevacizumab was used at half dose $(7.5 \mathrm{mg} / \mathrm{kg}$ ) with respect to the GOG-218 dose and therefore the optimal dose and schedule may warrant further debate.

The use of antiangiogenic drugs in the maintenance setting may give rise to various concerns on the basis of pre-clinical observations which indicate that inhibition of angiogenesis, even when exhibiting potent inhibitory effects in localised tumours, drives tumours to stages of greater malignancy 


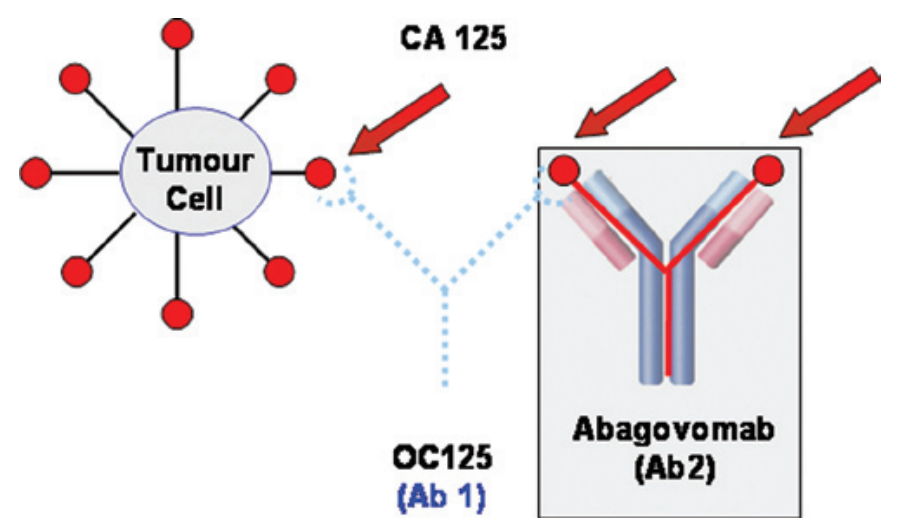

Figure 1. Injection of a tumour-associated antigen binding antibody (Ab1) leads to an immune response containing antibodies (Ab2) mimicking the structure of the original antigen.

increasing invasion and distant metastasis thus shortening the survival of mice (47-49). It has yet to be determined how general these results are and how they can be translated into the clinic for the best use of this class of drugs.

A further possibility for targeting ovarian cancer angiogenesis is represented by TK and mTOR inhibition. Two phase II trials are testing this hypothesis with sorafenib (www.clinicaltrial.gov; NCT00390611) and enzastaurin (www.clinicaltrial. gov; NCT0039111). Their results are expected to be presented within the next year and the findings may clarify the effectiveness of this approach, which in terms of feasibility provides certain advantages due to the oral administration of the two drugs.

EGFR has been found to be 'overexpressed' by the cells of numerous cancers, including ovarian, and may be considered an alternative target for the treatment of ovarian cancer. EGFR is reported to be present in ovarian cancers between 33 and $75 \%(50,51)$, and it has been implicated in both the growth and progression of this disease. Ovarian cancers that express increased concentrations of the EGFR are associated with poor survival $(50,52)$. Erlotinib, in a phase II trial in patients with recurrent ovarian cancer, showed a certain degree of activity with a manageable safety profile (53). Erlotinib, also an oral drug, is a good candidate for maintenance therapy.

A randomised phase III trial is assessing the efficacy of erlotinib compared to observation in patients who previously completed first-line chemotherapy for ovarian, peritoneal or fallopian tube cancer. This trial started in 2008, the enrollment has been completed, and the final results are expected within the next 12-24 months (www.clinicaltrials.gov; NCT00263822).

Immunoboosting approaches. Specific anti-cancer immunotherapy, in contrast to previous clinical findings, has demonstrated that in treated ovarian cancer patients it is possible to elicit a specific anti-cancer response. These results, even if controversial, offer the possibility that various regimens or products induce a more sustained response and finally a significant clinical benefit, thus rendering this field crucial. Oregovamab, which failed the phase III trial as a maintenance monotherapy post front-line chemotherapy, has recently shown that it is easily administered concomitantly with front-line chemotherapy. The combination is able to enhance specific CA125 cellular immunity, and preliminary clinical evidence favors this particular schedule (54). CA125 is also the target of the murine monoclonal antibody, abagovomab, a therapeutic vaccine in development as maintenance therapy for ovarian cancer patients (50).

The therapeutic strategy behind abagovomab is based on the 'immune network hypothesis' formulated by Niels Jerne (55). According to this theory, the variable antigen-binding regions of antibodies (Ab1) contain idiotypic determinants that are immunogenic and are able to induce anti-idiotypic antibodies (Ab2). A subset of these antibodies is able to functionally mimic the original antigen. Thus, immunization with anti-idiotypic antibodies could induce in the host a specific immune reaction (Ab3-mediated) directed against the original antigen.

In particular, abagovomab is an $\mathrm{Ab} 2$ antibody, which was obtained by immunizing mice with OC125, an antibody specific for CA125. In a subsequent phase, hybridoma cells, able to produce through a biotechnological process a large amount of abagovomab, were obtained (Fig. 1). Collectively, these characteristics make abagovomab different from the previous CA125-targeted antibody oregovomab, which directly binds the antigen; this gives abagovomab a stronger potential immunogenic activity.

To date, the results obtained in clinical trials support this mechanism of action. Abagovomab is capable of breaking the tolerance to CA125 in patients with CA125-expressing ovarian cancer, and the immunogenic response elicited in these patients is associated with prolonged survival.

In the first Phase Ib/II Proof of Concept (POC) study (56,57), 119 patients were treated with abagovomab after failure to respond to at least one previous chemotherapy. Vaccination of patients with abagovomab demonstrated induction of an anti-anti-idiotypic immune response (Ab3), in approximately $68 \%$ of the patients, which led to an anti-CA125 immune reaction (56). A specific cellular immune response was evidenced in a subgroup of these patients. Patients who produced a specific immune response after vaccination with abagovomab (68\%) showed an improved OS (23.4 months) as compared to those who did not (4.9 months). Moreover, the vaccination was well-tolerated, irrespective of the number of injections received.

To evaluate the effect of different doses (0.2 vs. $2 \mathrm{mg})$, schedules (6 vs. 9 vaccinations) and routes of administration (subcutaneous vs. intramuscular), abagovomab underwent two additional phase I/II studies that treated 78 patients overall $(58,59)$.

In these latter studies, the vaccinated patients developed a specific immune response, and the results allowed the selection of the dose ( $2 \mathrm{mg}$ ), the schedule (an injection every 2 weeks as induction during the first 6 weeks followed by monthly vaccination thereafter as maintenance), and the route of administration (subcutaneous) to be used in the pivotal efficacy trial.

In December 2006, a phase II-III trial, MIMOSA (www. mimosa-study.com), was initiated, and enrollment has been completed. The trial is a multicentre, double-blind, placebocontrolled study in patients with stage III-IV epithelial ovarian cancer who obtained a complete clinical response (evaluated 
by computer tomography and a CA125 value within the normal laboratory range) after debulking surgery and standard platinum/taxane first-line chemotherapy. Patients vaccinated with abagovomab as maintenance therapy, received a single subcutaneous injection every 2 weeks (for the first 4 doses induction phase) and then every 4 weeks (maintenance phase). Efficacy of abagovomab vs. placebo is primarily evaluated as increase in recurrence-free survival. Also, prolongation of life expectancy will be evaluated. The final results are expected in the first half of 2011. Preliminary blinded results on the safety and immune response of the 888 enrolled patients were reported at the last ASCO meeting, confirming that abagovomab administered as a repeated monthly administration is a safe and tolerable regimen, which induces an immune response comparable to the one observed in previous studies (Sabbatini et al: J Clin Oncol 28 (Suppl), abs. 5036 ASCO, 2010).

\section{Conclusions}

To date, only prolonged administration of taxol up to 12 cycles and the combination of bevacizumab plus chemotherapy followed by a long maintenance period of bevacizumab $12-15$ cycles at various doses $(15-7.5 \mathrm{mg} / \mathrm{kg})$ have demonstrated a statistical significant prolongation of PFS (6 and 3.8 months, respectively; data not known for a $7.5 \mathrm{mg} / \mathrm{kg}$ dose). However, the additional 12 taxol cycles did not have any impact on OS, while data for OS is still pending for bevacizumab trials. Both types of approach imply a long treatment duration that might affect the quality of life of patients. Moreover, the overall costs of bevacizumab treatments may be a further concern. In this context, the need for more active and feasible treatments remains high, and immunotherapy represents a valid option. Among 'immunostimulants' for the treatment of ovarian cancer, abagovomab is in the most advanced development stage. Furthermore, its optimal safety and tolerability profile demonstrated to date render it a noteworthy candidate for maintenance therapy in advanced ovarian cancer patients.

\section{References}

1. Bast RC, Hennessy B and Lills GB: The biology of ovarian cancer: new opportunities for translation. Nat Rev Cancer 9: 415-428, 2009

2. Yap TA, Carden CP and Kaye SB: Beyond chemotherapy: targeted therapies in ovarian cancer. Nat Rev Cancer 9: 167-181, 2009.

3. Menon U, Gentry-Maharaj A, Hallett R, et al: Sensitivity and specificity of multimodal and ultrasound screening for ovarian cancer, and stage distribution of detected cancers: results of the prevalence screen of the UK Collaborative Trial of Ovarian Cancer Screening (UKCTOCS). Lancet Oncol 10: 327-340, 2009.

4. Visintin I, Feng Z, Longton G, et al: Diagnostic markers for early detection of ovarian cancer. Clin Cancer Res 14: 1065-1072, 2008.

5. Yurkovetsky Z, Skates S, Lomakin A, et al: Development of a multimarker assay for early detection of ovarian cancer. J Clin Oncol 28: 2159-2166, 2010.

6. Kulasingam V, Pavlou MP and Diamandis EP: Integrating highthroughput technologies in the quest for effective biomarkers for ovarian cancer. Nat Rev Cancer 10: 371-378, 2010.

7. Paulsen T, Kjaerheim K, Kaern J, Tretli S and Trope C: Improved short-term survival for advanced ovarian, tubal, peritoneal cancer patients operated at teaching hospitals. Int J Gynecol Cancer 16: $11-17,2006$.

8. Engelen MJ, Kos HE, Willemse PH, et al: Surgery by consultant gynecologic oncologists improves survival in patients with ovarian carcinoma. Cancer 106: 589-598, 2006.
9. Earle CC, Schrag D, Neville BA, et al: Effect of surgeon speciality on processes of care and outcomes for ovarian cancer patients. J Natl Cancer Inst 98: 172-180, 2006.

10. Fung ET: A recipe for proteomics diagnostic test development: the OVA1 test, from biomarker discovery to FDA clearance. Clin Chem 56: 327-329, 2010.

11. Moore RG, McMeekin DS, Brown AK, et al: A novel multiple marker bioassay utilizing HE4 and CA125 for the prediction of ovarian cancer in patients with a pelvic mass. Gynecol Oncol 112: 40-46, 2009.

12. Ozols RF, Bundy BN, Greer BE, Fowler JM, Clarke-Pearson D and Burger RA: Phase III trial of carboplatin and paclitaxel compared with cisplatin and paclitaxel in patients with optimally resected stage III ovarian cancer: a Gynecologic Oncology Group study. J Clin Oncol 21: 3183-3195, 2003.

13. Dean M, Fojo T and Bates S: Tumor stem cells and drug resistance. Nat Rev Cancer 5: 275-284, 2005.

14. Szotek PP, Pieretti-Vanmarcke R, Masakiatos PT, et al: Ovarian cancer side population defines cells with stem cell-like characteristics and Mullarian inhibiting substance responsiveness. Proc Natl Acad Sci USA 103: 11154-11159, 2006.

15. Alvero AB, Chen $\mathrm{R}, \mathrm{Fu} \mathrm{H}$, et al: Molecular phenotyping of human ovarian cancer stem cells unravel the mechanisms for repair and chemo-resistance. Cell Cycle 8: 158-161, 2009.

16. Bapat SA, Mali AM, Koppikar CB and Kurrey NK: Stem and progenitor-like cells contribute to the aggressive behavior of human epithelial ovarian cancer. Cancer Res 65: 3025-3029, 2005.

17. Zhang S, Balch $\mathrm{C}$, Chan MW, et al: Identification and characterization of ovarian cancer-initiating cells from primary human tumors. Cancer Res 68: 4311-4320, 2008.

18. Baba T, Covery PA, Matsumura N, et al: Epigenetic regulation of CD133 and tumorigenicity of $\mathrm{CD} 133^{+}$ovarian cancer cells. Oncogene 28: 209-218, 2009.

19. Moserle L, Indraccolo S, Ghisi M, et al: The side population of ovarian cancer cells is a primary target of INF- $\alpha$ antitumor effects. Cancer Res 68: 5658-5668, 2008.

20. Hu L, McArthur C and Jaffe RB: Ovarian cancer stem-like side-population cells are tumourigenic and chemoresistant. Br J Cancer 102: 1276-1283, 2010.

21. Leizer AL, Alvero AB, Fu HH, et al: Regulation of inflammation by the NF- $\mathrm{KB}$ pathway in ovarian cancer stem cells. Am J Reprod Immunol: Sept. 5, 2010 (E-pub ahead of print).

22. Hall GD, Brown JM, Coleman RE, et al: Maintenance treatment with interferon for advanced ovarian cancer: results of the Northern and Yorkshire gynaecology group randomised phase III study. Br J Cancer 91: 621-626, 2004.

23. Widschwendter M, Fiegl H, Egle D, et al: Epigenetic stem cell signature in cancer. Nat Genet 39: 157-158, 2006.

24. Jones PA and Baylin SB: The epigenomic of cancer. Cell 128: 683-692, 2007

25. Ito K, Bernardi R and Pandolfi PP: A novel signalling network as a critical rheostat for the biology and maintenance of the normal stem cell and the cancer initiating cell. Curr Opin Genet Dev 19: 51-59, 2009

26. Park JT, Chen X, Tropè CG, Davidson B, Shih leM and Wang TL: Notch3 overexpression is related to the recurrence of ovarian cancer and confers resistance to carboplatin. Am J Pathol 177: 1087-1094, 2010.

27. Lis R, Touboul C, Mirshahi $\mathrm{P}$, et al: Tumor associated mesenchymal stem cells protects ovarian cancer cells from hyperthermia through CXCL12. Int J Cancer 128: 715-725, 2010.

28. Rothenberg ML, Liu PY, Wilczynski S, et al: Phase II trial of oral altretamine for consolidation of clinical complete remission in women with stage III epithelial ovarian cancer: A Southwest Oncology Group Trial (SWOG-9326). Gynecol Oncol 82: 317-322, 2001.

29. Bertelsen K, Jakobsen A, Stroyer I, et al: A prospective randomized comparison of 6 and 12 courses of cyclophosphamide, adriamycin, and cisplatin in advanced epithelial ovarian cancer: a Danish Ovarian Study Group trial (DACOVA). Gynecol Oncol 49: 30-36, 1993.

30. Lambert HE, Rustin GJ, Gregory WM and Nelstrop AE: A randomized trial of five versus eight courses of cisplatin or carboplatin in advanced epithelial ovarian carcinoma. Ann Oncol 8: 327-333, 1997.

31. Hakes TB, Chalas E, Hoskins WJ, et al: Randomized prospective trial of 5 versus 10 cycles of cyclophosphamide, doxorubicin, and cisplatin in advanced ovarian carcinoma. Gynecol Oncol 45: 284-289, 1992. 
32. Barakat RR, Sabbatini P, Bhaskaran D, et al: Intraperitoneal chemotherapy for ovarian carcinoma: results of long-term follow-up. J Clin Oncol 20: 694-698, 2002.

33. Markman M, Liu PY, Wilczynski S, et al: Phase III randomized trial of 12 versus 3 months of maintenance paclitaxel in patients with advanced ovarian cancer after complete response to platinum and paclitaxel-based chemotherapy: a Southwest Oncology Group and Gynecologic Oncology Group trial. J Clin Oncol 21: 2460-2465, 2003.

34. Perez-Gracia JL and Carrasco EM: Tamoxifen therapy for ovarian cancer in the adjuvant and advanced settings: systematic review of the literature and implications for future research. Gynecol Oncol 84: 201-209, 2002.

35. Berek J, Taylor P, McGuire W, Mary Smith L, Schultes B and Nicodemus CF: Oregovomab maintenance monoimmunotherapy does not improve outcomes in advanced ovarian cancer. J Clin Oncol 27: 418-425, 2009.

36. Ozols RF: Maintenance therapy in advanced ovarian cancer: progression-free survival and clinical benefit. J Clin Oncol 13: 2451-2453, 2003.

37. Wong C, Wellman TL and Lounsbury KM: VEGF and HIF-1 $\alpha$ expression are increased in advanced stages of epithelial ovarian cancer. Gynecol Oncol 91: 513-517, 2003.

38. Yamamoto S, Konishi I, Mandai M, et al: Expression of vascular endothelial growth factor (VEGF) in epithelial ovarian neoplasms: correlation with clinicopathology and patient survival, and analysis of serum VEGF levels. Br J Cancer 76 : 1221-1227, 1997

39. Brustamann $\mathrm{H}$ : Vascular endothelial growth factor expression in serous ovarian carcinoma: relationship with topoisomerase II alpha and prognosis. Gynecol Oncol 95: 16-22, 2004.

40. Kraft A, Weindel K, Ochs A, et al: Vascular endothelial growth factor in the sera and effusion of patients with malignant and non malignant disease. Cancer 85: 178-187, 1999.

41. Mesiana S, Ferrara N and Jaffe RB: Role of vascular endothelial growth factor in ovarian cancer: inhibition of ascite formation by immunoneutralization. Am J Pathol 153: 1249-1256, 1998.

42. Mabuchi S, Terai Y, Morishige K, et al: Maintenance treatment with bevacizumab prolongs survival in an in vivo ovarian cancer model. Clin Cancer Res 14: 7781-7789, 2008.

43. Burger RA, Sill MW, Monk BJ, Greer BE and Sorosky JI: Phase II trial of bevacizumab in persistent or recurrent epithelial ovarian cancer or primary peritoneal cancer: a Gynecologic Oncology Group Study. J Clin Oncol 25: 5165-5171, 2007.

44. Cannistra SA, Matulonis UA, Penson RT, et al: Phase II study of bevacizumab in patients with platinum-resistant ovarian cancer or peritoneal serous cancer. J Clin Oncol 25: 5180-5186, 2007.

45. Richardson DL, Backes FJ, Seamon LG, et al: Combination gemcitabine, platinum, and bevacizumab for the treatment of recurrent ovarian cancer. Gynecol Oncol 111: 461-466, 2008.

46. Kaye SB: Bevacizumab for the treatment of epithelial ovarian cancer: will this be its finest hour? J Clin Oncol 25: 5150-5152, 2007.
47. Pàez-Ribes M, Allen E, Hudock J, et al: Antiangiogenic therapy elicits malignant progression of tumors to increased local invasion and distant metastasis. Cancer Cell 15: 220-231, 2009.

48. Ebos JM, Lee CR, Cruz-Munoz W, Bjarnason GA, Christensen JG and Kerbel RS: Accelerated metastasis after short term treatment with a potent inhibitor of tumor angiogenesis. Cancer Cell 15: 232-239, 2009.

49. Loges S, Mazzone M, Hohensinner P and Carmeliet P: Silencing or fueling metastasis with VEGF inhibitors: angiogenesis revisited. Cancer Cell 15: 167-170, 2009.

50. Berchuck A, Rodriguez GC, Kamel A, et al: Epidermal growth factor receptor expression in normal ovarian epithelium and ovarian cancer. I. Correlation of receptor expression with prognostic factors in patients with ovarian cancer. Am J Obstet Gynecol 164: 669-674, 1991.

51. Morishige $\mathrm{K}$, Kurachi $\mathrm{H}$, Amemiya $\mathrm{K}$, et al: Involvement of transforming growth factor alpha/epidermal growth factor receptor autocrine growth mechanism in an ovarian cancer. Cancer Res 51: 5951-5955, 1991.

52. Bartlett JM, Langdon SP, Simpson BJ, et al: The prognostic value of epidermal growth factor receptor mRNA expression in primary ovarian cancer. Br J Cancer 73: 301-306, 1996.

53. Gordon AN, Finkler N, Edwards RP, et al: Efficacy and safety of erlotinib $\mathrm{HCl}$, an epidermal growth factor receptor (HER1/ EGFR) tyrosine kinase inhibitor, in patients with advanced ovarian carcinoma: results from a phase II multicenter study. Int J Gynecol Cancer 15: 785-792, 2005.

54. Braly $\mathrm{P}$, Nicodemus $\mathrm{CF}$, $\mathrm{Chu} \mathrm{C}$, et al: The immune adjuvant properties of front-line carboplatin-paclitaxel: a randomized phase 2 study of alternative schedules of intravenous oregovomab chemoimmunotherapy in advanced ovarian cancer. J Immunother 32: 54-65, 2009.

55. Jerne NK: Towards a network theory of the immune system. Ann Immunol 125C: 373-389, 1974.

56. Wagner U, Köhler S, Reinartz S, et al: Immunological consolidation of ovarian carcinoma recurrences with monoclonal anti-idiotype antibody ACA125: immune responses and survival in palliative treatment. Clin Cancer Res 7: 1154-1162, 2001.

57. Reinartz S, Köhler S, Schlebusch H, et al: Vaccination of patients with advanced ovarian carcinoma with the anti-idiotype ACA125: immunological response and survival (phase Ib/II). Clin Cancer Res 10: 1580-1587, 2004.

58. Pfisterer J, du Bois A, Sehouli J, et al: The anti-idiotypic antibody abagovomab in patients with recurrent ovarian cancer. A phase I trial of the AGO-OVAR. Ann Oncol 17: 1568-1577, 2006.

59. Sabbatini P, Dupont J, Aghajanian C, et al: Phase I study of abagovomab in patients with epithelial ovarian, fallopian tube, or primary peritoneal cancer. Clin Cancer Res 12: 5503-5510, 2006. 